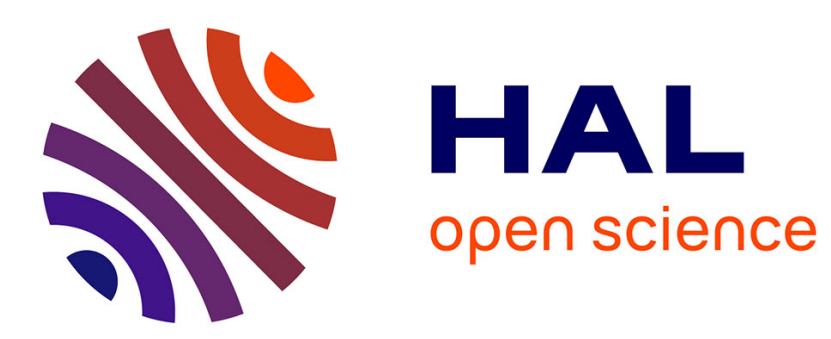

\title{
Identifying groups of critical edges in a realistic electrical network by multi-objective genetic algorithms
}

Enrico Zio, L. R. Golea, Claudio Rocco

\section{To cite this version:}

Enrico Zio, L. R. Golea, Claudio Rocco. Identifying groups of critical edges in a realistic electrical network by multi-objective genetic algorithms. Reliability Engineering and System Safety, 2012, 99, pp.172-177. 10.1016/j.ress.2011.11.008 . hal-00721479

HAL Id: hal-00721479

https://hal-centralesupelec.archives-ouvertes.fr/hal-00721479

Submitted on 27 Jul 2012

HAL is a multi-disciplinary open access archive for the deposit and dissemination of scientific research documents, whether they are published or not. The documents may come from teaching and research institutions in France or abroad, or from public or private research centers.
L'archive ouverte pluridisciplinaire HAL, est destinée au dépôt et à la diffusion de documents scientifiques de niveau recherche, publiés ou non, émanant des établissements d'enseignement et de recherche français ou étrangers, des laboratoires publics ou privés. 


\title{
Identifying groups of critical edges in a realistic electrical network by multi-objective genetic algorithms
}

\author{
E. Zio ${ }^{1,2}$, L.R. Golea ${ }^{2}$, C. M. Rocco S. ${ }^{3}$ \\ ${ }^{1}$ Ecole Centrale Paris- Supelec, France \\ ${ }^{2}$ Politecnico di Milano, Italy \\ ${ }^{3}$ Universidad Central de Venezuela, Caracas, Venezuela
}

\begin{abstract}
In this paper, an analysis of the vulnerability of the Italian high-voltage $(380 \mathrm{kV})$ electrical transmission network (HVIET) is carried out for the identification of the groups of links (or edges, or arcs) most critical considering the network structure and flow. Betweenness centrality and network connection efficiency variations are considered as measures of the importance of the network links. The search of the most critical ones is carried out within a multi-objective optimization problem aimed at the maximization of the importance of the groups and minimization of their dimension. The problem is solved by using a genetic algorithm. The analysis is based only on information on the topology of the network and leads to the identification of the most important single component, couples of components, triplets and so forth. The comparison of the results obtained with those reported by previous analyses indicates that the proposed approach provides useful complementary information.
\end{abstract}

Keywords: genetic algorithm, multi-objective optimization, network performance measure, power system vulnerability

\section{Introduction}

Societies are heavily dependent on many systems of distributed service, the so called infrastructures, e.g. computer and communication systems [Cohen et al., 2000], electric power transmission and distribution systems [Dobson et al., 2007], rail and road transportation systems [Zheng et al., 2007], constituted by networks of components. Such infrastructures are becoming more and more interconnected with each other, and therefore interdependent and potentially more vulnerable with respect to faults or malicious attacks: a random failure or an intentional attack in any of these complex networks may easily propagate across the network, increasing and multiplying its effects. 
These systems need priority protection, as specified by a number of national and transnational directives [CNIP, 2006], [Birchmeier, 2007]. The implementation of these directives and programs calls for the establishment of proper frameworks of vulnerability analysis for designing the adequate protections against failures and attacks [CNIP, 2006], [Rocco et al., 2007], [Vulnerability ESREL, 2007].

In order to protect a large-scale critical infrastructure, the Decision-Maker (DM) must be able to assess what elements are critical, that is what elements affect the system performance the most. From a topological viewpoint, various measures of the importance of a network element (edge or node), i.e., of the relevance of its location in the network with respect to a given topological indicator, can be introduced. The term 'importance' is then intended to qualify the role that the presence and location of the element plays with respect to the average global and local connection properties of the whole network.

In the case of electrical power systems, the existing literature on vulnerability analysis largely takes a topological approach to identify the critical components in the network [Albert et al., 2004], [Crucitti et al., 2005], [Zio et al., 2008]. Such analyses are capable of identifying elements of structural vulnerability, i.e. network edges and nodes whose failure can induce a severe structural damage to the network through the physical disconnection of its parts. Such analysis is very fast from a computational point of view and only requires the information of the topology of the network.

On the contrary, this kind of analysis is limited by the fact that it focuses only on the topological features of the network, thus neglecting its physical characteristics. In this respect, it is important to verify the extent of these limitations and possibly overcome them by additional more detailed physical analyses on critical parts of the network [Eusgeld et al., 2009], [Bompard et al., 2009], [Bier et al, 2007].

In this paper, an analysis of the vulnerability of the Italian high-voltage $(380 \mathrm{kV})$ electrical transmission network (HVIET) is carried out for the identification of the groups of elements most critical with respect to the network connection topology. In [Cadini et al., 2009], the authors proposed the use of betweenness centrality of groups of nodes as importance measures. In this paper, the approach is extended to consider the betweenness centrality of groups of edges and the variation in network connection efficiency [Latora and Marchiori, 2001] for identifying the critical groups. For the type of system under study this point of view is more realistic since in power systems, transmission lines (edges) are more exposed to attacks than nodes (substations).

The identification of the most critical groups of edges of different sizes in a network is a NPcomplete combinatorial problem, which may be effectively tackled by heuristic procedures, such as 
evolutionary search algorithms. In this paper, Genetic Algorithms (GAs) are used within a multiobjective formulation of the search problem, in which the decision variables are the groups of edges and the objectives are to maximize the importance of the groups, while minimizing their cardinality. This formulation guides the search towards the identification of the most important single components, couples of components, triplets and so forth. In other words, the problem is a multiobjective decision problem and the genetic algorithms are used to search for Pareto optimal solutions (i.e., non-dominated solutions): in this sense, several authors speak of multi-objective genetic algorithms, MOGA [Murata and Ishibuchi, 1995; Coello Coello et al., 2007].

The results are compared with those reported in [Rosato et al., 2006] and [Rocco et al., 2009]; in the first reference, the authors adopt a DC power flow to assess the behavior of the power system under a limited set of edges outages whereas in the second reference the most critical groups of edges are identified by evaluating the maximum flow in the power network, within a multiobjective formulation. Both papers try to model the network considering the physical phenomenon. In the case of [Rosato et al. 2006], they used a DC power flow, a simplified model that take into consideration the impedances of the network, the transmission line capacities and the load and generation nodes. In the approach proposed by [Rocco et al. 2009], a proxy of the power flow is used, by evaluating the maximum flow in the network: its value corresponds to the total load of the system. This approach requires in addition to the topology of the network, the capacity of the transmission lines, the load and generation nodes.

The paper is organized as follows. The next Section 2 presents the measures used to quantify the importance of groups of edges. Section 3 presents the proposed optimization approach. In Section 4, the approach is applied to the Italian high-voltage $(380 \mathrm{kV})$ electrical transmission network. Conclusions on the outcomes of the analysis are drawn in Section 5.

\section{Centrality measures}

The network system under analysis is modeled as a graph $G(V, E)$ where $V$ represents the set of vertexes (or nodes) ( $N=\operatorname{dim}(V)$ is the number of nodes) and $E$ represents the set of edges $(i, j)$ ( $K=\operatorname{dim}(E)$ is the number of edges). The graph is represented by its $N \times N$ adjacency (connection) matrix $\left\{a_{i j}\right\}$ whose entries are 1 if there is an edge joining nodes $i$ and $j, 0$ otherwise. Associated with each link $(i, j)$ is a $K \times K$ matrix $\left\{\gamma_{i j}\right\}$, describing the capacities of the links $(i, j)$. Additionally, a node is classified as a generating source (i.e., power source) or a load (i.e., power demand) or simply a junction node (neither generating nor demanding power). 
To evaluate the role played by the edges of the network with respect to its connectivity, two quantitative measures are considered in this paper.

\subsection{Group betweenness centrality measure}

Edge betweenness was first proposed by Girvan [Girvan and Newman, 2002] as a measure of centrality used to detect community structure in networks of various kinds. This measure is based on the idea that an edge is central if it is traversed by many of the shortest paths connecting pairs of nodes, from generation nodes to load nodes. Edge betweenness is a measure of the influence of an edge over the flow of information in the network, especially in cases where information flow follows the shortest available path. Note that this measure does not evaluate the magnitude of the flow through the shortest path.

By generalizing this concept, the betweenness centrality of a group of edges, denoted by $C^{B}(g)$, can be defined as follows:

$$
C^{B}(g)=\frac{\sum_{i, j \in G, i<j} \frac{s_{i, j}(g)}{s_{i, j}}}{(N-\operatorname{dim}(g)-1)(N-\operatorname{dim}(g))}
$$

where $g$ is the subset of edges of the graph, and $s_{i, j}(g)$ represents the number of geodesics (i.e., the shortest paths) connecting $i$ to $j$ that pass through $g$. In other words, the group betweenness centrality measure indicates the proportion of geodesics connecting pairs of non-group members that pass through the group. In the above equation, the sum is taken over all pair of nodes. The measure is normalized by dividing by the theoretical maximum value. If there is more than one shortest path between a pair of nodes, each path is given equal weight such that the total weight of all of the paths is unity. Note that all the information required to evaluate this measure is contained in the adjacency matrix of the network.

\subsection{Global efficiency relative variation importance}

The global efficiency of the graph $E_{f}(G)$ representing the network is defined as [Latora and Marchiori, 2001]:

$$
E_{f}(G)=\frac{1}{N(N-1)} \sum_{i, j \in G, i \neq j} \frac{1}{d i j}
$$

where $1 / d_{i j}$ is the efficiency of the connection between nodes $i$ and $j$ in terms of the number of edges on the shortest path linking the two nodes. It relates the importance of an edge to the impact on the network transmission performance of losing to failure the edges of a group. The relative 
variation of the global efficiency due to the removal of a group $g$ of edges is computed as the difference between the global efficiency of the network with all the edges of the group removed and the global efficiency of the original network, normalized to the latter value: $\left(E_{f}(G)-E_{f}\left(G^{\prime}\right) / E_{f}(G)\right)$; $G^{\prime}=G-g$. This value can be interpreted as a measure of importance of the group of edges removed [Crucitti et al., 2005].

As for the previous measure, all the information required to evaluate $E_{f}(G)$ is contained in the adjacency matrix of the network.

\section{Multi-objective genetic algorithm approach}

Genetic Algorithms (GAs) are powerful heuristics that have been successfully used in optimization and search problems. The terminology adopted in GAs contains many terms borrowed from biology, suitably redefined to fit the algorithmic context.

Thus, GAs operate on a set of (artificial) chromosomes, which are strings of numbers, generally sequences of binary digits (bits) 0 and 1, coding the values of the decision variables. The values of one or more objective functions in correspondence of the values of the decision variables of a chromosome, define the fitness of that chromosome. The GA search is performed by constructing a sequence of populations of chromosomes, the individuals of each population being the children of those of the previous population and the parents of those of the successive population.

The initial population is generated by randomly sampling the bits of all the strings; at each step in the search sequence, the new population is obtained by probabilistically manipulating the strings of the old population with fitness-improving rules, which mimic genetic evolution. The search sequence continues until a pre-established optimality termination criterion is reached. The string manipulation consists in selecting and mating pairs of chromosomes in order to groom chromosomes of the next population. This is done by repeatedly performing on the strings the four fundamental operations of reproduction, crossover, replacement and mutation, all based on random sampling: the parents selection step determines the individuals which participate in the reproduction phase; reproduction itself allows the exchange of already existing genes whereas mutation introduces new genetic material; the substitution defines the individuals for the next population. This way of proceeding enables to efficiently arrive at optimal or near-optimal solutions. Since the initial population is generated at random, it is important to perform several runs in order to assess the convergence of the heuristic. As with any other heuristic, the approach cannot be claimed to converge for every problem setting. 
In the case of a multiobjective optimization problem, two or more possibly conflicting objective functions $f_{i}(\cdot), i=1,2, \ldots, n_{f}$, must be evaluated in correspondence with each decision variable vector $U$ in the search space. In this case, the GA search proceeds by comparing the solutions in terms of the concepts of Pareto optimality and dominance [Goldberg, 1989]. The decision variable vectors which are not dominated by any other of a given set are called non-dominated with respect to this set while the decision variable vectors that are non-dominated within the entire search space are said to be Pareto optimal and constitute the so called Pareto optimal front, which is the object of the optimization.

\subsection{Genetic Algorithm for identifying critical groups}

Consider a network $G(V, E)$ and one of the measure defined by (1) or (2). Let

$$
C\left(x_{1}, x_{2}, \ldots, x_{K}\right)
$$

represent the importance measure selected when different edges are grouped, with $x_{i}=1$ if edge $i$ belongs to the group, $x_{i}=0$ otherwise. For example, in a network with $K=3$ edges and using (1), $C(1,0,0)$ indicates the betweenness importance of the first edge alone; $C(0,0,1)$ indicates the importance of the third edge alone; $C(1,0,1)$ indicates the betweenness importance of the group made of the first and third edges.

In a network with $K$ edges, the number of groups (single edges, pairs, triplets and so forth) that in principle can be formed is $2^{K}$. A complete analysis of all groups to find the most critical is therefore impractical for large networks [Zio and Golea, 2009]. This fact suggests devising appropriate heuristic procedures to solve the problem, as the one based on GA here proposed.

To overcome this obstacle, the task of determining the most critical groups of edges can be framed as a multiobjective (MO) optimization problem with respect to the two following objectives:

$$
\begin{gathered}
\underset{x}{\operatorname{Max}} f_{1}(x)=C\left(x_{1}, x_{2}, \ldots, x_{K}\right) \\
\underset{x}{\operatorname{Min}} f_{2}(x)=\sum_{i=1}^{K} x_{i}
\end{gathered}
$$

with $x_{i}=1$ if edge $i$ belongs to the group, $x_{i}=0$ otherwise; thus $f_{2}(x)$ represents the cardinality of the group of edges.

The need for introducing the second objective is due to the fact that, in general, the more edges in a group, the higher its impact on the network. Indeed, a search directed only by the importance of the 
groups (with no control on their sizes) would favor large groups, whose simultaneous failure or attack (in a security view) is however not likely (on the contrary, this is not so within cascading processes which can lead to large number of edges failing successively).. By defining the second objective, which favors small groups, the search is guided to finding the most critical (i.e., $\left.\max f_{1}(x)\right)$, preferably small-size groups, i.e., the single edges, pairs, triplets and so forth.

Note that alternatively one could introduce the limit on the group size to a certain number, as a constraint of the single-objective minimization of eq. (4). Fixing the group size to a specific value as constraint would require analyzing the effects of different group sizes by repeating several times the single-objective problem solution with varying values of the constraint; on the contrary, the multi-objective approach by the introduction of the second objective allows us to obtain Paretooptimal solutions in a single run.

In most typical applications of GAs, every proposal of solution, represented by the vector $x$ of the independent decision variables, is coded in a chromosome constituted by genes, each one coding one decision variable of $x$. Differently from typical applications, the decision variables $\left(x_{1}, x_{2}, \ldots, x_{K}\right)$ of eq. (4) of the optimization problem considered in this paper are already in the form of a binary string; therefore, single-gene chromosomes are used, directly coding the composition of the group. The dimension of the chromosome is equal to the number of edges in the network. Table 1 shows two examples of chromosome-coding for a system of $K=10$ edges.

Table 1. Examples of chromosome-coding of groups of edges in a network of 10 edges

\begin{tabular}{|l|l|l|l|l|l|l|l|l|l|l|}
\multicolumn{7}{|c|}{ Chromosome coding (10 bits) } & \multicolumn{1}{|c|}{$\begin{array}{l}\text { Corresponding edges in } \\
\text { the group }\end{array}$} \\
\hline 1 & 2 & 3 & 4 & 5 & 6 & 7 & 8 & 9 & 10 & \\
\hline 0 & 0 & 0 & 1 & 0 & 0 & 1 & 0 & 1 & 0 & $4,7,9$ \\
\hline 1 & 0 & 0 & 0 & 0 & 1 & 0 & 1 & 0 & 1 & $1,6,8,10$ \\
\hline
\end{tabular}

\section{The Italian high-voltage $(380 \mathrm{kV})$ electrical transmission network}

The Italian high-voltage $(380 \mathrm{kV})$ electrical transmission network (HVIET) can be represented by an undirected graph $G(V, E)$ with $N=310$ nodes and $K=361$ edges (Figure 1). Three different types of nodes can be distinguished: source nodes $S$ (where power is inserted in the network), load nodes $L$ (where power is extracted from the network) and junction nodes $J$ (which are neither $S$ nor $L$ nodes). There are $S=97$ source nodes, $L=113$ load nodes and $J=100$ junction nodes. Some 
edges (14 over 361) are constituted by double lines; for the topological analysis carried out in this work, double lines are considered as providing a single edge of communication.

Several topological properties have been analyzed on the HVIET network [Crucitti et al., 2004], [Tiriticco et al., 2006]. A more detailed analysis of the vulnerability has been performed by [Rosato et al., 2006], based on a model reproducing the power flow conditions of the network. The obtained results showed that "only a small number of nodes having a "functional" relevance for the network can be discovered through the topology analysis of its graph". Finally, the authors remarked that "topological analysis and the simulation of the "functional" models (such as the DC power flow model, for the case of electrical networks) provide complementary information".

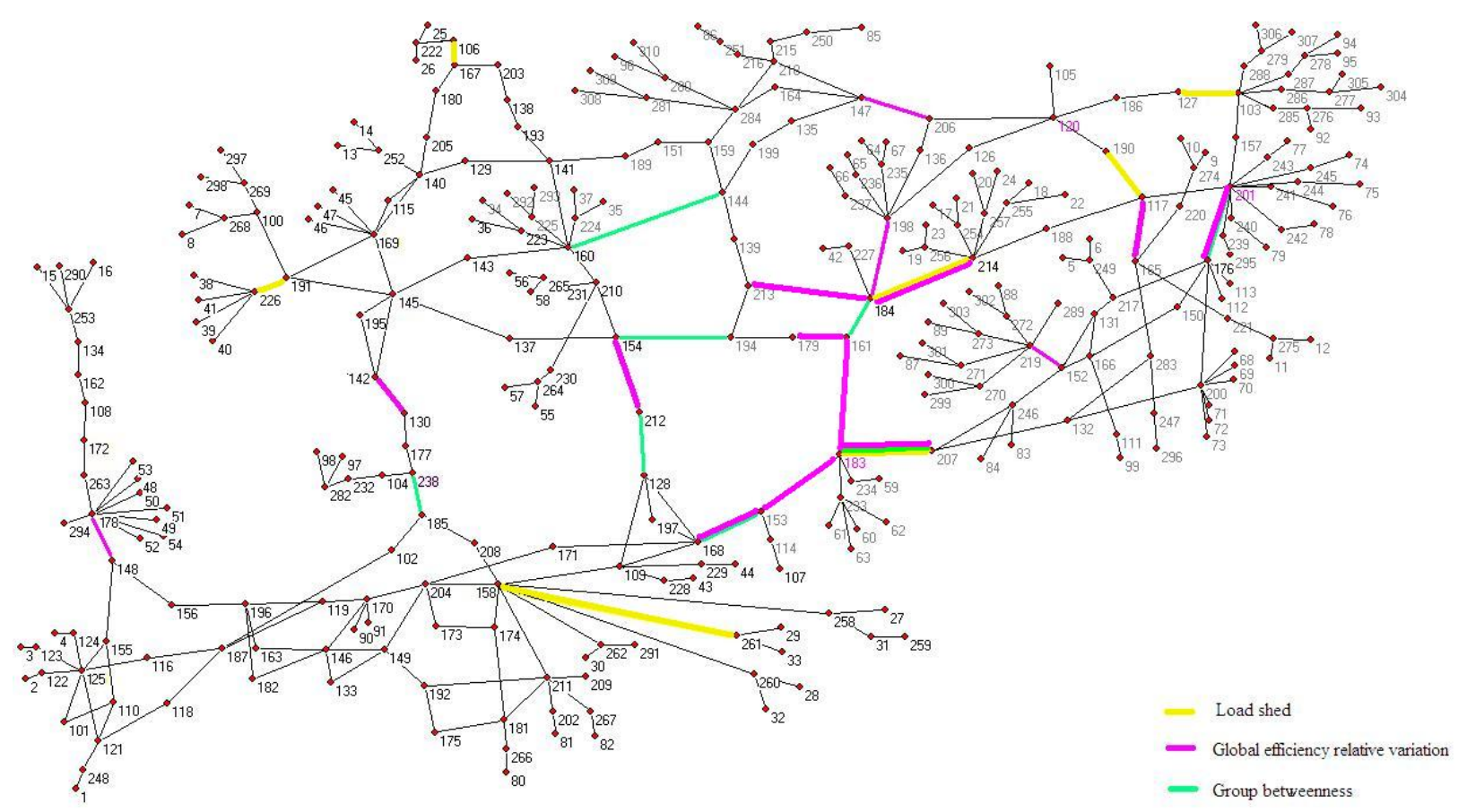

Figure 1. The Italian high-voltage transmission network, drawn using Pajek [Batagelj and Mrvar, 1998]

A set of GAs has been implemented to identify the most critical groups of edges of different sizes in the network of Figure 1. Table 2 summarizes the details of the implementation of the GA operators previously described, along with a number of parameters that control the operation of the genetic algorithms such as the population size (i.e., the size of the evolving set of candidate solutions) and the number of generations (i.e., the duration of the evolution process). Five GA optimization runs were performed for identifying the critical groups with respect to betweenness centrality and five with respect to global efficiency relative variation importance, starting from different initial random populations. The number of solutions found at the end of each search was around 5-7 (i.e., 5-7 
different group sizes, from 1 to 5-7). A Pareto set was then obtained by selecting the non-dominated solutions among those reached in the different runs.

Table 2: Multi-objective genetic algorithm parameters and rules

\begin{tabular}{|l|c|}
\hline Number of chromosomes & 200 \\
\hline Number of generations & 800 \\
\hline Selection & Fit-Fit \\
\hline Replacement & Children-parents \\
\hline Mutation probability & 0.001 \\
\hline Crossover probability & 1 \\
\hline $\begin{array}{l}\text { Number of generations without elitist } \\
\text { selection }\end{array}$ & 0.25 \\
\hline $\begin{array}{l}\text { Fraction of parents chosen with elitist } \\
\text { selection }\end{array}$ & 15 \\
\hline
\end{tabular}

Table 3 provides the most vulnerable edges in the Pareto set found by the genetic algorithm MO search driven by the global efficiency relative variation: the edges 184-214 and 183-207, identified as critical for electrical transport, are also present in the groups of size five. Figure 2 shows the Pareto front approximation. Each point in the graph represents a group of critical edges.

The drop in efficiency due to the removal of the two edges, individually, is $-2.3 \%$ and $-3.9 \%$; when they are both removed, the drop is $-7.6 \%$, which confirms the importance of analyzing groups of elements. The rest of edges forming the groups of the Pareto set are located at the boundaries of the north side of the network, which is indicated as the most vulnerable part of the network system.

Table 4 reports the most important groups of edges identified with respect to the betweenness centrality measure. The results show that the removal of the edges with high betweenness leads to the separation of groups from one another. Note that for the network under study, the most important groups contain edges that are physically concentrated in the central part of the network; failures of these divide the network in separate, isolated parts. 
Table 3. Pareto optimal results of the multi-objective search for global efficiency relative variation and the corresponding groups betweenness values

\begin{tabular}{|c|c|c|c|c|c|c|c|c|c|}
\hline $\begin{array}{l}\text { Global efficiency } \\
\text { relative variation - } \\
\text { Pareto }\end{array}$ & $\begin{array}{l}\text { Group } \\
\text { Size }\end{array}$ & \multicolumn{7}{|c|}{ Edges out (from-to node) } & \multirow{2}{*}{$\begin{array}{c}\begin{array}{c}\text { Group } \\
\text { betweenness }\end{array} \\
0.687\end{array}$} \\
\hline$-49.5 \%$ & 7 & $165-117$ & $148-178$ & $154-212$ & $130-142$ & $153-168$ & $176-201$ & $161-183$ & \\
\hline$-46.8 \%$ & 6 & $165-117$ & & $154-212$ & $130-142$ & $153-168$ & $176-201$ & 161-183 & 0.652 \\
\hline$-38.7 \%$ & 5 & & $148-178$ & & $183-207$ & $184-214$ & $184-198$ & $147-206$ & 0.577 \\
\hline$-34.7 \%$ & 4 & & $153-183$ & 161-179 & & & $184-213$ & $206-147$ & 0.505 \\
\hline$-29.6 \%$ & 3 & & $153-183$ & $154-212$ & $130-142$ & & & & 0.442 \\
\hline$-12.6 \%$ & 2 & $152-219$ & $148-178$ & & & & & & 0.194 \\
\hline$-6.6 \%$ & 1 & $152-219$ & & & & & & & 0.086 \\
\hline
\end{tabular}

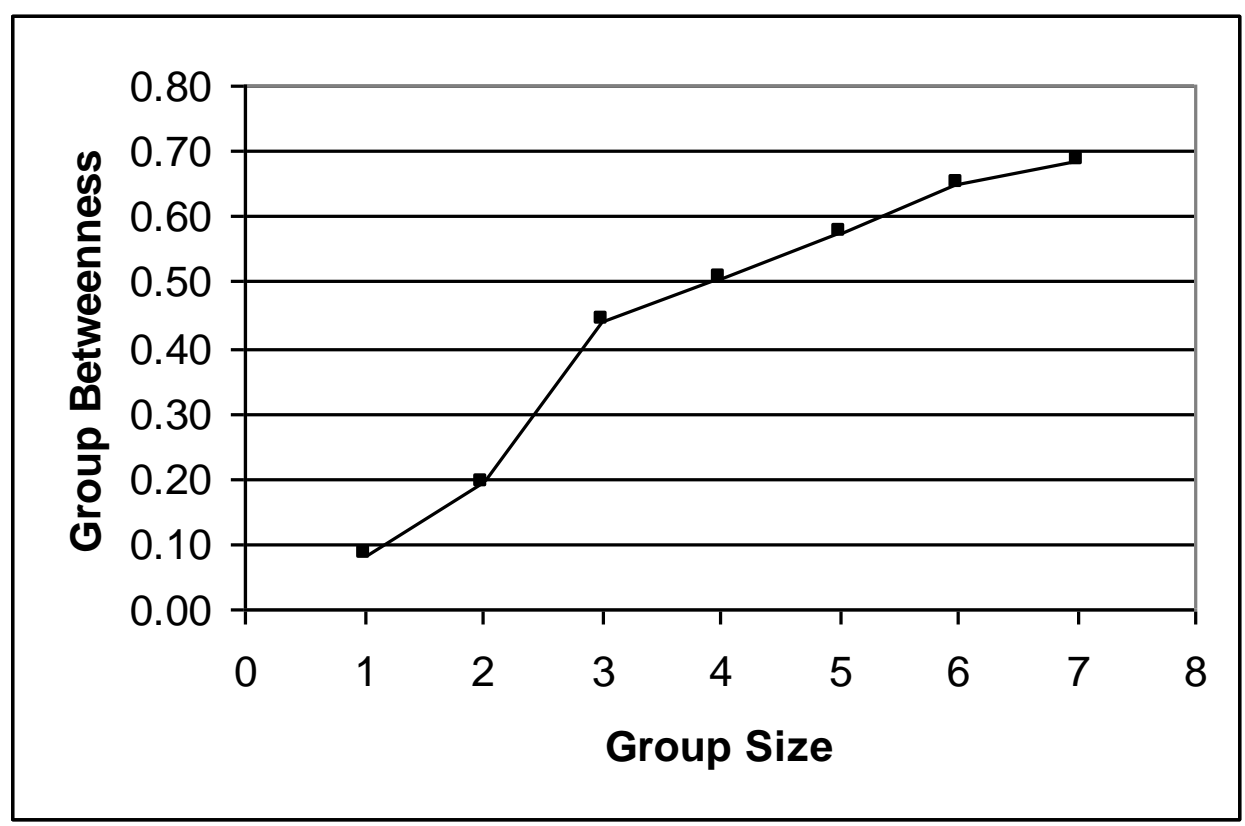

Figure 2. Pareto front approximation: Group Size vs Betweenness

Table 4. Pareto optimal results of the multi-objective search for betweenness centrality groups and values of the efficiency variation of the corresponding edges 


\begin{tabular}{|c|c|c|c|c|c|c|c|c|c|}
\hline $\begin{array}{c}\text { Group } \\
\text { betweenness } \\
\text { Pareto }\end{array}$ & $\begin{array}{c}\text { Group } \\
\text { size }\end{array}$ & \multicolumn{7}{|c|}{ Edges out (from-to node) } & $\begin{array}{l}\text { Global efficiency } \\
\text { relative variation }\end{array}$ \\
\hline 0.758 & 7 & $153-168$ & 144-160 & 154-194 & $128-212$ & 117-201 & $185-238$ & 183-207 & $-35.8 \%$ \\
\hline 0.707 & 6 & $153-168$ & $144-160$ & 154-194 & $128-212$ & \begin{tabular}{|l|}
$176-201$ \\
\end{tabular} & $185-238$ & & $-33.9 \%$ \\
\hline 0.65 & 5 & $153-168$ & $144-160$ & 154-194 & $128-212$ & $176-201$ & & & $-16.8 \%$ \\
\hline 0.584 & 4 & $153-168$ & $144-160$ & 154-194 & $128-212$ & & & & $-15 \%$ \\
\hline 0.504 & 3 & $153-168$ & $144-160$ & 154-194 & & & & & $-12.7 \%$ \\
\hline 0.384 & 2 & $153-168$ & $144-160$ & & & & & & $-4.9 \%$ \\
\hline 0.258 & 1 & $153-168$ & & & & & & & $-3.7 \%$ \\
\hline
\end{tabular}

As a term of comparison, the detailed approach presented in [Rosato et al., 2006] recognizes that the edges critical for electrical transport are those connecting nodes 103, 117, 127, 184, 190 and 214; these edges also form a subset of the screening results obtained in [Rocco et al., 2009] (Table 5, columns 1-3). The results summarized in Table 5 show that the most critical groups identified in [Rocco et al., 2009] in terms of load shed are assembled around the group of edges 184-214, 183207, 103-127, 117-190. These edges are concentrated on the north-east side of the network in Figure 1, with edges 184-214 and 183-207 in the center of this area which has turned out to be the most vulnerable part of the network system according to the group measures considered in the multiobjective GA search. A significant drop in efficiency $(-32.2 \%$, Table 5 , column 5 , of a maximum of $-34.7 \%$, Table 3, column 1) was obtained in the case of interdiction of the edges composing the most important group of size four in terms of load shed, leading to the conclusion that the failure of the edges affects the network both from a structural and functional point of view. The global efficiency relative variation results of Table 5 show a significant drop of the efficiency of the network for the most critical groups identified, of size four to seven. This leads to the conclusion that the removal of specific edges redirects the flow towards the shortest topological paths.

Table 5. Pareto optimal results of the multiobjective search, and corresponding group betweenness and global efficiency relative variation 


\begin{tabular}{|c|c|c|c|c|c|c|c|c|c|c|}
\hline $\begin{array}{l}\text { Load } \\
\text { Shed- } \\
\text { Pareto }\end{array}$ & $\begin{array}{c}\text { Group } \\
\text { Size }\end{array}$ & \multicolumn{7}{|c|}{ Edges out (from-to node) } & \multirow{2}{*}{$\begin{array}{c}\text { Group } \\
\text { betweenness } \\
0.52\end{array}$} & \multirow{2}{*}{\begin{tabular}{|c|}
$\begin{array}{c}\text { Global } \\
\text { efficiency } \\
\text { relative } \\
\text { variation }\end{array}$ \\
$-36.9 \%$
\end{tabular}} \\
\hline $26 \%$ & 7 & $106-167$ & $103-127$ & 184-214 & $183-207$ & $117-190$ & $158-261$ & $191-226$ & & \\
\hline $23 \%$ & 6 & $106-167$ & $103-127$ & $184-214$ & $183-207$ & $117-190$ & $158-261$ & & 0.49 & $-34.9 \%$ \\
\hline $21 \%$ & 5 & $106-167$ & $103-127$ & $184-214$ & $183-207$ & $117-190$ & & & 0.47 & $-33.4 \%$ \\
\hline $16 \%$ & 4 & & $103-127$ & $184-214$ & $183-207$ & $117-190$ & & & 0.45 & $-32.2 \%$ \\
\hline $12 \%$ & 3 & & $103-127$ & $184-214$ & $183-207$ & & & & 0.42 & $-8.4 \%$ \\
\hline $8 \%$ & 2 & & $103-127$ & $184-214$ & & & & & 0.16 & $-2.8 \%$ \\
\hline $4 \%$ & 1 & 106-167 & & & & & & & 0.025 & $-1.5 \%$ \\
\hline
\end{tabular}

Finally, the outcomes of the analysis of the critical groups of edges are qualitatively similar to those obtained in [Rosato et al., 2006]: a limited number of "central" edges with "functional" relevance for the network is discovered through the topological analysis of its graph. Also, the most critical groups of edges identified in [Rocco et al., 2009] in terms of the load shed and those identified in this paper with respect to the edge betweenness expand around a core of edges, which can be subject to further analysis by simulation of AC/DC load flow.

On the other hand, differences in the results exist due to the different model assumptions driving the two models. In [Rocco et al., 2009], the maximum flow reduction is used as performance function for carrying out an analysis which is a proxy of the physical analysis with additional information requirements. The search is done aiming at the minimum set of edges to interdict for maximizing the load shed. Note that the group of size four could be considered a fourth-order cut set for the north-east side of the network. In this view, the reason why the critical group search performed in this paper does not identify edges $117-190$ as critical is explained by the fact that these nodes are high-generation capacity nodes and as such they are identified as critical in [Rocco et al., 2009]: on the contrary, the measures (1) and (2) driving the criticality search performed in this paper do not consider capacity. 


\section{Conclusions}

In this paper, a GA-based procedure for the vulnerability analysis of a network system has been devised to identify the most critical groups of edges of different sizes in the network. The GA search for the most important groups has been framed as a multi-objective optimization problem whose decision variables are the edge group compositions and the objectives are the maximization of the importance of the groups and the minimization of group cardinality.

The Italian high-voltage electrical transmission network (HVIET) has been taken as case study for the analysis of the importance of groups of edges considering the network structure and flow. The group betweenness centrality measure and the global efficiency relative variation have been used as importance indicators to underline the structural vulnerabilities. The results have been compared with the results of two approaches previously presented in the literature, obtained from a more realistic functional vulnerability analysis of the network: one based on the evaluation of the load flow through a DC load flow model; the other using the maximum flow in the network, as a proxy of the real load flow. It has been shown that the proposed approach requires minimum information of the network (only the topology of the network) and leads to the identification of a number of critical edges that have 'functional' relevance and provides complementary information, which could be used as a preliminary screening analysis.

As a general remark, the proposed procedure allows for a preliminary analysis for identifying groups of critical edges by analyzing only the topology of the network. This requires a minimum of information. The analysis produces a set of critical elements that must be analyzed by appropriate procedures that model the physical phenomenon of the network. The results suggest that a preliminary set of critical edges can be provided by the proposed approach. For obtaining realistic insights on the robustness and vulnerability of realistic electrical transmission systems to faults and attacks, the analysis focused on the topological features of the network must be expanded to include also additional physical characteristics. In this respect, work is currently undergoing in establishing effective ways of bringing different physical characteristics into the topological analysis, e.g. the reliability and electrical characteristics of the transmission edges in a power network.

\section{References}

[Albert et al., 2004] Albert, R., Albert, I., Nakarano, G.L., Phys.Rev.E, 69, 025103(R), 2004.

[Batagelj and Mrvar, 1998] Batagelj, V., Mrvar, A., Pajek - program for large network analysis, Connections, 21, 47-57, 1998. 
[Bier et al, 2007] Bier, V.M., Gratz, E.R., Haphuriwat, N.J., Magua, W. and Wierzbicki, K.R. (2007). Methodology for identifying near-optimal interdiction strategies for a power transmission system. Reliability Engineering \& System Safety, 92(9), pp.1155-1161.

[Birchmeier, 2007] Birchmeier J., Systematic Assessment of the Degree of Criticality of Infrastructures, Proceedings of ESREL 2007, Stavanger, Norway, Vol. 1, pp. 859-864, 25-27 June 2007.

[Cadini et al, 2009] Cadini F., Zio E. Petrescu C.A.: Using Centrality Measures to Rank the Importance of the Components of a Complex Network Infrastructure, Lecture Notes in Computer Science, 2009, Volume 5508/2009, 155-167

[CNIP, 2006] CNIP'06, Proceedings of the International Workshop on Complex Network and Infrastructure Protection, Rome, Italy, 28-29 March 2006.

[Coello Coello, 2007] Coello Coello C.A., Lamont G.A., Van Veldhuizen D.A.: Evolutionary Algorithms for Solving Multi-Objective Problems Second Edition, Springer, 2007

[Cohen et al., 2000] Cohen, R., Erez, K., ben-Avraham, D., Havlin, S., Resilience of the Internet to random breakdowns, Physical Review Letters 85(21): 4626-4628, 2000.

[Crucitti et al., 2005] Crucitti, P., Latora, V., Marchiori M., Locating critical lines in high-voltage electrical power grids, Fluct. Noise Lett., 5, L201-L208.

[Crucitti et al., 2004] Crucitti, P., Latora, V., Marchiori M., A Topological Analysis of the Italian Electric Power Grid, Physica A Vol. 338, pp. 92-97, 2004.

[Eusgeld et al., 2009] Eusgeld, I., Kroger, W., Sansavini, G., Schlapfer, M., Zio, E., The role of network theory and object-oriented modeling within a framework for the vulnerability analysis of critical infrastructures, Reliability Engineering \& System Safety, Vol. 94, Issue 5, pp 954-963, May 2009.

[Dobson et al., 2007] Dobson, I., Carreras, B.A., Lynch, V.E., Newman, D.E., Complex systems analysis of series of blackouts: Cascading failure, critical points, and self-organization, Chaos: An Interdisciplinary Journal of Nonlinear Science 17(2): 026103, 2007.

[Ford and Fulkerson, 1962] Ford, L. and Fulkerson, D., Flows in Networks, Princeton University Press, Princeton, NJ, 1962.

[Girvan and Newman, 2002] Girvan, M., Newman, M. E. J., Community structure in social and biological networks, Proc. Natl. Acad. Sci., USA 99, 5890, 2002.

[Goldberg, 1989] Goldberg D.E., Genetic algorithms in search, optimization, and machine learning, Addison-Wesley Publ. Co, 1989.

[Latora and Marchiori, 2001] Latora V. and Marchiori M., Efficient Behavior of Small-World Networks, Physical Review Letters, Vol. 87, N. 19, 2001. 
[Murata and Ishibuchi, 1995]Murata, T., Ishibuchi, H.: MOGA: multi-objective genetic algorithms, IEEE International Conference on Evolutionary Computation, 1995, pp 289

[Rocco et al., 2007] Rocco, C.M., Zio, E., Salazar, D.E., Multi-objective Evolutionary Optimisation of the Protection of Complex Networks Exposed to Terrorist Hazard, Proceedings of ESREL 2007, Stavanger, Norway, Volume 1, pp. 899-905, 25-27 June 2007.

[Rocco et al., 2009] Rocco, C.M., Ramirez-Marquez, J.E., Salazar, D.E., Zio, E., A Flow Importance Measure via Multiple-Objective Optimization and its Application to an Italian Transmission Power System, accepted for publication in International Journal of Performability Engineering, 2009.

[Rosato et al., 2006] Rosato, V., Issacharoff, L., Bologna, S., Influence of the topology on the power flux of the Italian high-voltage electrical network, Europh. Lett., 2006.

[Tiriticco et al., 2006] Tiriticco, F., Bologna, S., Rosato, V., Electr. Power Syst. Res., 2006.

[Vulnerability ESREL, 2007] Vulnerability, Reliability and safety of Complex Networks and Critical Infrastructures, Special Sessions I and II, Proceedings of ESREL 2007, Stavanger, Norway, Volume 1, 25-27 June 2007.

[Zheng et al., 2007] Zheng, J.-F., Z.-Y. Gao, Zhao, X.-M., Clustering and congestion effects on cascading failures of scale-free networks, EPL (Europhysics Letters)(5): 58002, 2007.

[Zio et al., 2006] Zio, E., Baraldi, P., Pedroni, N. Feature selection for Transients Classification by A Niched Pareto Genetic Algorithm. in: D. Ruan, P. D’Hondt, P. Fantoni, M.D. Cock, M. Nachtegael, E.E. Kerre (Eds.), Applied Artificial Intelligence: Proceeding of the 7th International Flins Conference: 938-945, 2006.

[Zio et al., 2008b] Zio, E., Petrescu, C.A., Sansavini, G., Vulnerability analysis of a power transmission network, Proceedings of PSAM9 - International Probabilistic Safety Assessment and Management Conference, Hong Kong, China, 18-23 May 2008.

[Zio and Golea, 2009] Zio, E., Golea, L.R., Identification of betweenness-central groups of components in a complex network infrastructure by genetic algorithms, accepted, ESREL Safety and Reliability for Managing Risk, Prague, Czech Republic, 7-10 September 2009. 